\title{
Utilización del Value Efficiency Analysis (VEA) en la medición de la eficiencia en la atención primaria $^{*}$
}

\author{
Eduardo González Fidalgo \\ Universidad de Oviedo \\ Vicente García González \\ Gerencia de Atención Primaria, Área Sanitaria V \\ Servicio de Salud del Principado de Asturias
}

\section{Resumen}

El Análisis Envolvente de Datos (DEA) ha sido una técnica muy utilizada para evaluar la eficiencia de la actividad sanitaria, debido a su carácter conservador y su elevada flexibilidad. Sin embargo, la aplicación empírica de esta metodología no está exenta de importantes dificultades. Un conocido y recurrente problema del análisis DEA es su escaso poder discriminante cuando el número de dimensiones es elevado pero la muestra disponible no lo es. Este trabajo explora las posibilidades de incrementar el poder discriminante del DEA mediante una extensión llamada Value Efficiency Analysis (VEA). El VEA exige incorporar información de carácter cualitativo para identificar una unidad productiva que pueda considerarse un buen referente global. Al aplicar la técnica sobre una muestra de 61 centros de salud asturianos se comprueba la mejora de la capacidad discriminante, al reducirse en un $32 \%$ el número de centros de salud considerados eficientes. Los resultados del análisis evidencian un problema de escala en algunos centros de salud de gran tamaño. Asimismo, se detecta una notable relación entre la ineficiencia y el porcentaje de población anciana asignada al centro de salud. En cambio, no se encuentra relación con la docencia MIR ni con el nivel de dispersión geográfica.

Palabras clave: eficiencia, DEA, VEA, primaria, centros de salud, Asturias Clasificación JEL: C63, D24, I12

\section{Resumen}

Data Envelopment Analysis (DEA) has been extensively employed in evaluating the productivity of health services due to its enormous flexibility and the conservative character of its results. However, the empirical application of DEA frequently faces important problems. A well known drawback of DEA is its low discriminating power when the number of dimensions is large but the sample at hand is not. This paper explores the possibility to improve the discriminating power of DEA using a refinement known as Value Efficiency Analysis (VEA). VEA incorporates some qualitative information in order to identify a

* El presente trabajo ha sido realizado con una ayuda para Proyectos de Investigación del FIS, expediente N. ${ }^{\circ} 04 / 0942$.

Los autores desean hacer constar su agradecimiento a las Gerencias de Atención Primaria del SESPA por haber facilitado amablemente los datos que lo han hecho posible y a los Técnicos de Salud de las diferentes Áreas Sanitarias por su intermediación a la hora de conseguirlos.

El trabajo se ha beneficiado enormemente de los útiles comentarios realizados por Ángel López Nicolás a versiones previas del mismo. 
decision making unit that may be considered as a good referent globally. The application of this technique to a sample of 61 primary health centers in Asturias shows a strong improvement in the discriminating power, obtaining a reduction of $32 \%$ in the number of centers that are considered efficient. The results also evidence a scale problem in some of the biggest health centers. We find a notable relationship between productive efficiency and the percentage of aged population that is assigned to the health center. In contrast, there is no significant relationship between efficiency and teaching (MIR) or geographical dispersion.

Key words: efficiency, DEA, VEA, primary health centers, Asturias

JEL Classification: C63, D24, I12

\section{Introducción}

La medición de la eficiencia en el ámbito sanitario es siempre un proceso complejo, debido especialmente a la dificultad para valorar adecuadamente el output sanitario (Fuentelsaz, Marcuello y Urbina, 1996). Considerando el nivel de salud de la población como indicador ideal nos encontramos con que, además de las dificultades para establecer un consenso sobre lo que se entiende por salud, ésta puede verse afectada por variables externas a las estrictamente sanitarias, como el medio ambiente, la dotación genética y biológica o los estilos de vida. Por ello, es común trabajar con outputs intermedios como los indicadores clásicos de gestión de forma que se identifican no un único ouput sino varios, en correlato con un número también variable de inputs (Fuentelsaz, Marcuello y Urbina, 1996; Urbina, 1997).

Esta situación se acentúa especialmente en el caso de la Atención Primaria, por sus características propias y por el papel que juega en el contexto de los diferentes actores que intervienen en la asistencia sanitaria. En este ámbito, resulta especialmente difícil identificar un producto específico y propio de la Atención Primaria, más allá de los indicadores clásicos de número de consultas, presión asistencial o frecuentación. Sin embargo, estos indicadores son insatisfactorios en la medida en que no está clara la relación entre un mayor número de consultas y una mejora en la salud de la población, por lo que no sería adecuado hablar de eficiencia en términos de volumen de producción exclusivamente (Puig, 2000; Pinillos, 2003).

Como forma de superar este obstáculo se han diseñado indicadores relacionados con la calidad asistencial, como el tiempo medio por paciente, la satisfacción de la población, el grado de cobertura de la atención sanitaria para diversos servicios y colectivos de pacientes o de poblaciones o el cumplimiento de las normas técnicas (de calidad) de la cartera de servicios (Pujol et al, 2006).

En el caso de la Cartera de Servicios se ha utilizado un indicador sintético, el Producto de la Cartera de Servicios (Alonso et al, 1995) que indica la cobertura ponderada de la población a la que se prestan los diferentes servicios de la Cartera. Este indicador cuantitativo se completa con la cumplimentación de las normas técnicas (de calidad) de los diferentes servicios, lo que introduce también un aspecto cualitativo en la medición del producto. Sin embargo, este planteamiento ha sido criticado por insuficiente (ya que no cubre todos los aspectos propios de la Atención Primaria) e insa- 
tisfactorio (Pinillos, 2003), a pesar de lo cual resulta la aproximación más cercana a lo que sería una idea del producto (en términos de proceso) de la Atención Primaria. Otros indicadores, como los de resultado, aún están en fase de planeamiento y desarrollo para lo que deberán solventar también sus dificultades de medida. Propuestas como los Ambulatory Care Groups, pese a haber sido ya validados, no terminan de implantarse, seguramente como consecuencia de las dificultades existentes para su aplicación a la práctica (García et al, 1998; Juncosa et al., 1999; Orueta et al., 1999).

En el lado de los inputs, al lado de los indicadores clásicos como el gasto farmacéutico o de personal, se han introducido también en los Contratos de Gestión pactos acerca del número de pruebas diagnósticas o de interconsultas (derivaciones) con especialistas. Otras cuestiones, como el coste de la Incapacidad Transitoria (IT) y las relacionadas con la imputabilidad de ese gasto, como la prescripción inducida, aunque de interés, resultan de difícil incorporación y valoración.

Finalmente, se ha valorado la eficiencia a través de ratios que establecen relaciones entre un determinado output y un determinado input. El problema es que no existe una única ratio que mida la eficiencia, ya que como hemos expuesto existen múltiples inputs para producir uno o varios outputs. A ello habría que añadir que no siempre los indicadores van en la misma dirección, con lo que en ocasiones resulta difícil establecer donde está el nivel óptimo de la ratio utilizada como indicador de valoración (Goñi, 1998). En este contexto resulta de interés disponer de una herramienta que permita transformar una situación productiva en la que diversos recursos generan múltiples productos en un único índice de eficiencia.

El análisis DEA se ha utilizado extensamente en el ámbito sanitario (Puig, 2000; Puig y Dalmau, 2000), resultando un procedimiento adecuado por su facilidad para tener en cuenta simultáneamente las diferentes dimensiones del output sanitario (en cantidad y calidad). No obstante, el DEA adolece de una escasa capacidad discriminante, especialmente cuando se tienen en cuenta muchas dimensiones de inputs y outputs (Ali, 1994). En esos casos, los resultados muestran muchas unidades como eficientes por el mero hecho de ser incomparables en alguna dimensión con el resto de la muestra (utilizar la menor cantidad de un input, por ejemplo). Algunos avances recientes en la metodología DEA permiten atenuar la importancia de este problema, a costa de una mayor complejidad analítica.

El objetivo de este trabajo es evaluar la eficiencia de los centros de salud de Asturias por comparación de su actividad, utilizando medidas tanto de cantidad como de calidad. Se toma como punto de partida el trabajo anterior de García y González (2004) que analizó la ineficiencia de una submuestra de centros de salud de Asturias mediante la técnica de análisis envolvente de datos (DEA). En este trabajo se empleará una metodología basada en el DEA, que añade una restricción sobre las ponderaciones. Esta técnica, denominada VEA (Value Efficiency Analysis), permite incrementar el poder discriminante del DEA al incorporar información sobre una unidad productiva que pueda considerarse como una referencia en cuanto a su combinación de factores y productos. Asimismo, el trabajo pretende examinar los factores que se relacionan con el nivel de eficiencia VEA. Para ello, el artículo se estructura de la si- 
guiente manera. En el Apartado 2 se expone la metodología VEA como una extensión del tradicional DEA. El Apartado 3 presenta los datos utilizados en el análisis. A continuación se resumen los resultados de las estimaciones en el Apartado 4. Por último, se recogen las principales conclusiones derivadas del estudio.

\section{Métodos}

En primer lugar utilizaremos la metodología de análisis envolvente de datos (DEA) para obtener una primera frontera de referencia para los centros de salud. Esta frontera nos permitirá encontrar los centros de salud que pueden considerarse eficientes desde el punto de vista técnico, es decir, los que se sitúan sobre la frontera y no por debajo de ella. Aunque existen numerosas variantes de los programas DEA, en este trabajo partimos de la especificación original de los programas de Charnes, Cooper y Rhodes (1978) para la frontera con rendimientos constantes a escala (CRS) y Banker, Charnes y Cooper (1984) para la frontera con rendimientos variables (VRS). Adoptamos un enfoque de maximización del output producido por la unidad. El modelo DEA con CRS y orientación output requiere resolver para cada unidad productiva $i$ el siguiente programa matemático:

$$
\begin{aligned}
& \min _{\frac{\sum_{m=1}^{M} v_{m} x_{i m}}{\sum_{s=1}^{S} u_{s} y_{i s}}}^{\text {s.a.: }} \\
& \frac{\sum_{m=1}^{M} v_{m} x_{j m}}{\sum_{s=1}^{S} u_{s} y_{j s}} \geq 1, \forall j \\
& u_{s}, v_{m} \geq 0, \forall s, m
\end{aligned}
$$

donde $x_{i m}$ representa la cantidad consumida por la unidad $i$ del input $m, y_{i s}$ representa la cantidad producida del output $s$ por la unidad $i, v_{m}$ es el precio sombra del input $m$ y $u_{s}$ el precio sombra del output $s$. El programa busca el conjunto de precios que hacen mínimo el coste de producción de la unidad i con respecto al valor de su producto, condicionado a que con esos precios todas las unidades obtengan un ratio mayor o igual que 1. Si la unidad $i$ es eficiente se obtendrán precios que den el mínimo valor posible del índice, es decir, la unidad, mientras que si es ineficiente se obtendrán valores superiores. El programa fraccional anterior presenta problemas para su cómputo, de manera que resulta más sencillo resolver el siguiente programa lineal equivalente. 


$$
\begin{aligned}
& \min \sum_{m=1}^{M} v_{m} x_{i m} \\
& \text { s.a.: } \\
& \sum_{s=1}^{S} u_{s} y_{i s}=1 \\
& \sum_{s=1}^{S} u_{s} y_{j s}-\sum_{m=1}^{M} v_{m} x_{j m} \leq 0, \forall j \\
& u_{s}, v_{m} \geq 0, \forall s, m
\end{aligned}
$$

De manera análoga, este programa busca los precios sombra para los que el coste de la unidad i es mínimo, normalizando el valor de los outputs en la unidad. Si la unidad $i$ es eficiente obtendrá un coste igual a 1, mientras que si es ineficiente obtendrá un coste mayor que uno, existiendo otra unidad en la muestra que obtiene un coste igual a 1 con los precios sombra más favorables para la unidad $i$. Este mismo programa se resuelve para todas las unidades de la muestra, obteniendo cada una de ellas sus precios sombra más favorables y sus correspondientes índices de eficiencia relativa. A efectos de presentación de resultados se suele transformar este índice por su inverso, de manera que valores menores a 1 indican el grado de ineficiencia productiva.

Si se relaja la condición CRS y se sustituye por el supuesto VRS, de mayor realismo, será necesario modificar el programa matemático anterior de la siguiente manera (Banker, Charnes y Cooper, 1984):

$$
\begin{aligned}
& \min \sum_{m=1}^{M} v_{m} x_{i m}+e_{i} \\
& \text { s.a.: } \\
& \sum_{s=1}^{S} u_{s} y_{i s}=1 \\
& \sum_{s=1}^{S} u_{s} y_{j s}-\sum_{m=1}^{M} v_{m} x_{j m}-e_{i} \leq 0, \forall j \\
& u_{s}, v_{m} \geq 0, \forall s, m
\end{aligned}
$$

donde se añade el término independiente $e_{i}$ para relajar la condición de que la función objetivo tenga que pasar por el origen, lo que implicaría CRS. Solo cuando $e_{i}$ tome el valor 0, la unidad evaluada se encontrará localmente en la zona de CRS.

La comparación entre el índice obtenido bajo el supuesto CRS y el índice obtenido bajo el supuesto VRS puede interpretarse como el efecto de la ineficiencia de escala, debida a un tamaño inadecuado de la unidad productiva. Por tanto, una medida de la ineficiencia de escala vendría dada por: 


$$
E S=\frac{E F_{C R S}}{E F_{V R S}}
$$

Una de las principales críticas que puede hacerse a la aproximación DEA es su enorme flexibilidad a la hora de seleccionar las ponderaciones o precios sombra de cada una de las unidades. Dado que el programa se resuelve de manera individual para cada unidad productiva, los precios sombra pueden no tener nada que ver entre diferentes unidades. La justificación de esta flexibilidad se basa en buscar una evaluación de la ineficiencia en el supuesto más benevolente para cada unidad evaluada. No obstante, tal supuesto puede parecer excesivo ya que provoca que las valoraciones de inputs y outputs sean completamente diferentes entre unidades. Para evitar este problema se han sugerido diversas fórmulas para restringir las ponderaciones en los programas DEA (Dyson y Thanassoulis, 1988; Allen, Athanassopoulos, Dyson y Thanassoulis, 1997; Roll, Cook y Golany, 1991; Wong y Besley, 1990; Pedraja, Salinas y Smith, 1997).

El principal problema a la hora de restringir directamente las ponderaciones es que implica realizar juicios de valor sobre el rango de precios sombra que se consideran adecuados. Halme, Joro, Korhonen, Salo y Wallenius (1999) han propuesto una metodología alternativa llamada Value Efficiency Analysis (VEA) capaz de restringir las ponderaciones incorporando información aportada por un experto. La gran aportación del VEA consiste en que en lugar de establecer los rangos en los que se tienen que mover los precios sombra, se pide al experto que seleccione una de las unidades eficientes como referente óptimo de comparación $(o)$. De esta manera, el programa introduce una nueva restricción consistente en que con los pesos de la unidad evaluada $(i)$ el referente absoluto u óptimo (o) debe obtener un índice de eficiencia igual a 1. Es decir, se exige que los precios sombra de la unidad evaluada sean adecuados para la unidad elegida como referente. En otras palabras, se exige a todas las unidades que sus precios sombra sean razonables para la unidad tomada como referente. Un efecto inmediato de esta restricción adicional en el programa DEA es que unidades que se consideraban eficientes por tener una estructura de outputs o inputs extrema, dejarán de serlo.

El programa VEA con VRS y orientación output presenta la siguiente especificación:

$$
\begin{aligned}
& \min \sum_{m=1}^{M} v_{m} x_{i m}+e_{i} \\
& \text { s.a.: } \\
& \sum_{s=1}^{S} u_{s} y_{i s}=1 \\
& \sum_{s=1}^{S} u_{s} y_{j s}-\sum_{m=1}^{M} v_{m} x_{j m}-e_{i} \leq 0, \forall j \\
& \sum_{m=1}^{M} v_{m} x_{o m}+e_{i}-\sum_{s=1}^{S} u_{s} y_{o s}=0 \\
& u_{s}, v_{m} \geq 0, \forall s, m
\end{aligned}
$$


donde se ha añadido la restricción de que la unidad tomada como referencia $(o)$ obtenga una eficiencia igual a 1 con los precios sombra de la unidad evaluada $(i)$. Al introducir esta restricción en todas las unidades, se restringe el rango de precios sombra admisibles al rango que admita la unidad de referencia $(o)$.

La Figura 1 ilustra la diferencia entre el DEA y el VEA para el caso de una tecnología con 2 outputs donde se mantienen constantes las cantidades de los inputs. Un análisis DEA mostraría que las 5 unidades A, B, C, D y E son eficientes, puesto que todas ellas se sitúan sobre la frontera de posibilidades de producción. Sin embargo, la unidad A toma precios sombra muy diferentes a los de la unidad E, para justificar su eficiencia. Concretamente pondera mucho más el valor del output $y_{2}$ que el valor del output $y_{1}$. La unidad $\mathrm{E}$ hace exactamente lo contrario. En muchos contextos productivos esta disparidad de precios sombra no puede justificarse. En el ámbito sanitario, por ejemplo, no sería aceptable que un centro de salud justificase su actividad productiva basándose en que el precio sombra de una consulta es 3 veces superior al de una visita domiciliaria y, al mismo tiempo, otro centro de salud justificase su actividad sobre la base de que una visita domiciliaria tiene un precio sombra 3 veces su-

FIGURA 1

VEA VS DEA

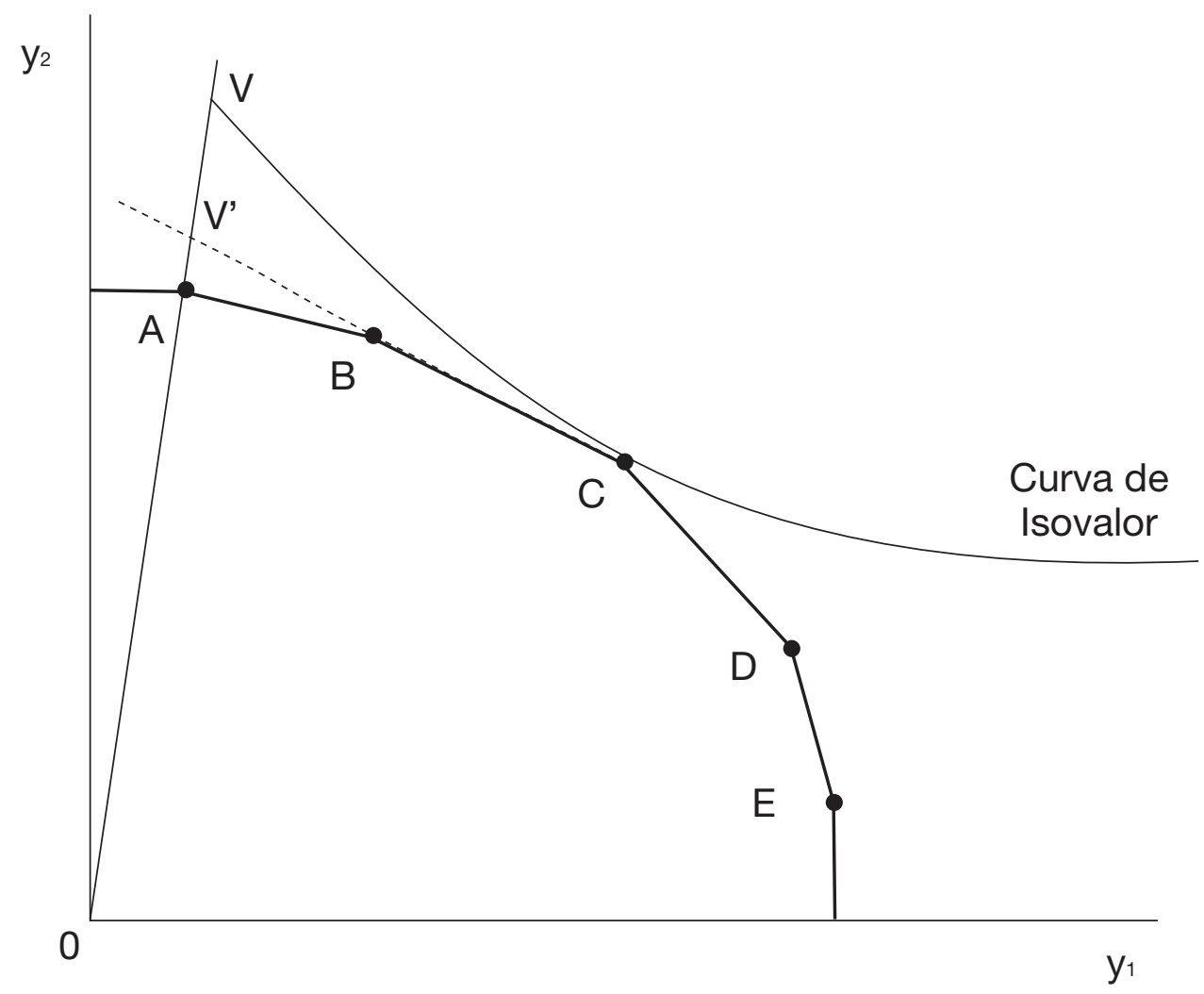


perior al de una consulta. Si se deja que cada centro de salud pondere más aquellas actividades en las que producen un mayor volumen o consumen menores cantidades de recursos, obtendremos resultados de eficiencia cuyas justificaciones son contradictorias entre sí.

El VEA exige elegir una unidad que debe permanecer eficiente bajo cualquier precio sombra que tomemos para valorar la eficiencia de las otras unidades. Por ejemplo, en la Figura 1 se ha tomado la unidad $\mathbf{C}$ como referencia. Teóricamente la actividad de la unidad C sería la que alcanzara la máxima curva de isovalor (por eso la selecciona el experto). La unidad A, por tanto, alcanza una curva de isovalor inferior y podemos medir su nivel de eficiencia como OA/OV. Esto indicaría el incremento en outputs necesario para alcanzar la misma curva de isovalor que la unidad tomada como referencia, C. Dado que las curvas de isovalor son desconocidas, el VEA propone una aproximación lineal, representada por la línea punteada. La eficiencia VEA de la unidad A se aproximaría por el cociente OA/OV'. Continuando con el ejemplo gráfico, puede comprobarse fácilmente que las unidades $\mathrm{B}$ y $\mathrm{D}$ seguirán siendo consideradas eficientes bajo la aproximación VEA, mientras que la unidad E será considerada ineficiente.

\section{Datos}

El estudio se ha realizado sobre el conjunto de los 61 centros de salud que, cumpliendo los criterios de inclusión, pertenecen a las 8 Áreas Sanitarias del Servicio de Salud del Principado de Asturias (SESPA). La muestra incluye Equipos o Unidades de Atención Primaria de las Zonas Básicas de Salud correspondientes a las Áreas de Asturias, entendiendo como tales aquellas unidades que firman Contrato Programa o de Gestión con la Gerencia y están formadas por varias unidades básicas de atención (médico más enfermera), independientemente de que dispongan de pediatría, y tienen la responsabilidad de prestar atención sanitaria a alguna de las zonas básicas que configuran el Mapa Sanitario de Asturias. Los consultorios periféricos se consideraron englobados dentro de los E.A.P. de los que dependían, contribuyendo con sus datos de actividad al conjunto de los aportados por la Unidad.

Al utilizar una técnica de evaluación que actúa por comparación es necesario que los términos que se comparan tengan un alto grado de homogeneidad (Pinillos, 2003). Con el fin de homogeneizar la muestra, se excluyen las Unidades de Apoyo y los Equipos o Unidades responsables de la cobertura de Zonas Especiales de Montaña. De igual forma se excluyeron del estudio aquellas unidades que todavía prestan atención mediante el modelo tradicional de asistencia. La información fue facilitada por las Gerencias de Atención Primaria de las Áreas que participaron en el estudio, a partir de sus registros de actividad y memorias. Los datos corresponden a un período de tiempo delimitado entre el 1 de enero de 2002 a 31 de diciembre de 2002 (ejercicio año 2002).

En el contexto de la actividad sanitaria no es sencillo establecer el conjunto de inputs y outputs que deben tenerse en cuenta a la hora de realizar el análisis (Pinillos, 2003). 
En nuestro caso consideraremos un conjunto de 4 indicadores de output y 4 de input. Varios estudios han considerado como producto de los centros de salud el valor de su cartera de servicios (Puig, 2000; García et al., 1999), definida por el conjunto de servicios que se ofrecen a la población adscrita al centro.

Esta variable se define como el porcentaje de la población adscrita que recibe cada uno de los servicios de la cartera (cobertura) ponderado por la carga de trabajo que el servicio representa para el profesional (INSALUD 1997, García y Minué 1998). Así, la cobertura de un Servicio viene dada por la relación existente entre las personas incluidas en Programa de dicho Servicio, respecto a las personas susceptibles de recibir dicho Servicio, es decir, respecto a la población diana del Servicio en cuestión. Para efectuar la ponderación por la carga de trabajo se utiliza un valor de consenso (valor técnico), establecido originalmente en el ámbito del extinto INSALUD por un grupo de expertos, que intenta reflejar la carga de trabajo que supone cada servicio, en términos tanto de captación como de seguimiento, tomando como valor unitario de referencia el del Servicio de vacunación de la gripe (INSALUD 1997, García et al., 1997).

El producto derivado de la multiplicación del valor técnico de cada servicio por su cobertura, determina el valor de lo que se denomina primer componente de la Cartera de Servicios. El valor final, para el conjunto de los Servicios incluidos en la Cartera, vendrá dado por la relación entre la suma de todos los productos respecto del mayor valor posible que se obtendría si todas las coberturas fueran del $100 \%$. El resultado se expresa como un porcentaje. En nuestro estudio, con el fin de transformar los valores de esta variable, un porcentaje, en otra de naturaleza más cuantitativa que la exprese en términos de volumen de población total cubierta por algún servicio de la cartera, se ha multiplicado este índice por la población de referencia del E.A.P. correspondiente. Matemáticamente podría expresarse:

$$
\frac{\sum_{i=1}^{I} \frac{n_{i}}{N_{i}} V T_{i}}{100 \cdot \sum_{i=1}^{I} V T_{i}} \cdot P
$$

donde,

$n_{i}$ : Total de casos o paciente incluidos en el servicio $i$

$N_{i}$ : Total de casos o paciente esperados en el servicio $i$

$V T_{i}$ : Valor Técnico asignado al servicio $i$

$P$ : $\quad$ Total de población asignada o bajo cobertura sanitaria del EAP

Además, se ha incluido como output el número total de consultas (como suma aritmética de las efectuadas por los Médicos de Familia, Pediatras y Enfermeros). En este sentido, se podría discutir la pertinencia de la inclusión de un output cuyo incremento no es necesariamente deseable, aunque aparente ser eficiente, ya que un mayor número de consultas médicas sobre una misma población con unos recursos de- 
terminados, podría implicar un descenso del tiempo por visita y, por ello, un descenso de la calidad con queta se presta la atención. Sin embargo la situación inversa podría suceder en el caso de las consultas de enfermería, en las que si existe un margen para su aumento sin por ello deteriorar la calidad de la asistencia. En consecuencia, dado que el balance entre lo que deberían disminuir unas (para mejorar la calidad) y aumentar las otras (para realizar más actividad), es positivo hacia un incremento del total en el número de consultas realizadas en los centros de atención primaria, se ha optado por reflejar este output como la suma aritmética de las mismas. Por otro lado, la mayor parte de los trabajos sobre eficiencia en centros de salud consideran las consultas como un output, a veces incluso el único output (Pinillos y Antoñanzas, 2002; Fuentelsaz, Marcuello y Urbina, 1996; Urbina y Serrano, 1997; Martí y Grenzner, 1999; García et al., 1996).

Finalmente, se han considerado entre los outputs el número de visitas domiciliarias de enfermería y el grado de cumplimentación de las normas técnicas de la Cartera de Servicios. Se pretende con este último output incluir una variable que aproxime el nivel de calidad en la prestación del servicio, ya que un mayor cumplimiento de las normas técnicas indica un mayor nivel de calidad.

En cuanto a la definición y valoración de los recursos (inputs) utilizados en la producción de los servicios la mayoría de los autores introducen los recursos humanos como una de las variables a tener en cuenta en el análisis de la eficiencia. Aunque la formalización de la variable podría admitir varias expresiones, como por ejemplo el gasto de personal a través del Capítulo I, en nuestro estudio hemos considerado como input el número total de efectivos (número de médicos, enfermeras, pediatras, y otro personal) en cada E.A.P.

Sin embargo, el factor trabajo no es el único determinante del producto de la atención primaria. Si excluimos las decisiones de inversión, que, al estar centralizadas, no se encuentran dentro de los márgenes de actuación de los centros de salud ${ }^{1}$, sí tendría sentido analizar aquellos gastos cuyo ahorro pueda contribuir a mejorar la eficiencia de los centros de salud. Esto justifica considerar entre los recursos el gasto en bienes corrientes (Capítulo II) y farmacia (Capítulo IV).

Finalmente, fueron considerados como inputs recursos que, provenientes de otros niveles del sistema sanitario, prestan funciones de apoyo en el proceso de diagnóstico y tratamiento. Este es el caso de las derivaciones para radiología, laboratorio (analíticas) e interconsulta de especialidades, como total de derivaciones (suma aritmética).

Como factor asociado con el nivel de eficiencia, ante la falta de disponibilidad, en este nivel asistencial, de indicadores relacionados con características relevantes de la población atendida (como la severidad, el diagnóstico o el motivo de consulta), se ha optado por el porcentaje de ancianos de la población adscrita a cada unidad. Puede considerarse como un indicador indirecto de casuística y determinante muy importante del gasto y del consumo de recursos. Como indicador indirecto de dispersión geográfica y de su carácter rural o urbano se ha elegido el valor $\mathrm{G}$ de la zona bá-

\footnotetext{
Además, no se constatan diferencias importantes en el equipamiento de los distintos centros.
} 
sica (a mayor valor G, mayor dispersión y ruralidad). Por último, como indicador del nivel de capital intelectual y gestión del conocimiento se ha optado por la docencia MIR en la especialidad de Medicina Familiar y Comunitaria. La Tabla 1 muestra las variables empleadas en el estudio.

\section{TABLA 1}

VARIABLES UTILIZADAS EN EL TRABAJO

\begin{tabular}{|c|c|c|}
\hline Inputs & Outputs & Variables relacionadas \\
\hline Recursos Humanos & Total consultas & Docencia (MIR) \\
Gastos corrientes (C II) & Visitas domiciliarias & Dispersión geográfica (G) \\
de enfermería & \% Población anciana (ANC) \\
Total derivaciones & Población*Cartera de & servicios \\
& Cumplimiento de Normas & \\
& Técnicas Mínimas & \\
\hline
\end{tabular}

\section{Resultados}

La Tabla 2 muestra el resumen de los índices de eficiencia DEA calculados para los centros de salud de la muestra con el modelo especificado en el apartado anterior. La tabla incluye una descripción de la eficiencia calculada sobre la frontera con CRS, la frontera con VRS y la eficiencia de escala (ES). Estos resultados indican una eficiencia técnica bastante alta en los centros de salud evaluados, puesto que la eficiencia media global $\left(\mathrm{EF}_{\mathrm{CRS}}\right)$ se sitúa en torno al $90 \%$. En cuanto a las causas de la ineficiencia, los resultados muestran una mayor ineficiencia debida a la escala $(93,6)$ y una menor responsabilidad de la ineficiencia debida a la gestión $(95,8)$. De hecho, el principal problema de ineficiencia de escala parece deberse a la presencia de rendimientos decrecientes en los centros de salud de mayor tamaño, puesto que de todos los centros que presentan ineficiencia de escala, solamente uno lo hace por operar con rendimientos crecientes, mientras que todos los demás están operando en la zona de rendimientos decrecientes.

TABLA 2

ESTADÍSTICOS DESCRIPTIVOS DE LA EFICIENCIA DEA

\begin{tabular}{|l|c|c|c|c|c|}
\hline & Media & SD & Mín. & N eficiente & \% eficiente \\
\hline $\mathbf{E F}_{\text {CRS }}$ & 89,8 & 9,9 & 67,5 & 19 & 31,1 \\
$\mathbf{E F}_{\text {VRS }}$ & 95,8 & 7,0 & 72,2 & 35 & 57,4 \\
$\mathbf{E S}$ & 93,6 & 6,8 & 73,9 & 19 & 31,1 \\
\hline
\end{tabular}


La tabla también muestra el número de centros de salud que se sitúan sobre la frontera, siendo este un número muy elevado, especialmente cuando se utiliza la frontera VRS. En ese caso, el 57\% de los centros de salud analizados pertenecen a la frontera. Esta circunstancia, evidencia el escaso poder discriminante del análisis DEA cuando el número de dimensiones (inputs+outputs) es elevado, en relación con el número de unidades contenidas en la muestra.

Si tomamos los resultados ofrecidos por el DEA deberíamos realizar una valoración muy positiva de la eficiencia en los centros de salud del Principado de Asturias. Con la excepción de algunos centros cuyos índices de eficiencia se sitúan por debajo del $80 \%$, la mayor parte de los centros presentan niveles de eficiencia muy altos, 1 de cada 3 no presenta ningún tipo de ineficiencia y 1 de cada 2 no muestra ninguna ineficiencia debida a una inadecuada gestión. No obstante, como se ha señalado anteriormente en este trabajo, el análisis DEA permite una flexibilidad excesiva para que cada centro «escoja» los precios sombra de inputs y outputs que le resulten más favorables, pudiendo existir una considerable disparidad de precios sombra entre las distintas unidades. Para evitar este problema y conseguir una mayor racionalidad en los precios sombra asignados por cada centro, decidimos seleccionar de entre los centros eficientes a uno que representara un comportamiento adecuado en todos los aspectos. Esta elección puede basarse en el criterio de un experto o en un análisis de los resultados DEA. En nuestro caso, se seleccionó como referente el centro número 3 por ser el que figura un mayor número de veces como referente de los centros ineficientes en el análisis DEA realizado anteriormente. Se trata de un centro eficiente que además sirve como referente de 26 de los centros considerados como ineficientes. Asimismo, el conocimiento personal del centro nos permite realizar una valoración subjetiva muy favorable de dicho centro de salud.

La restricción que añade el VEA consiste en que con los precios sombra de todos los centros evaluados, el centro número 3 tiene que conseguir una eficiencia del 100\%. Es decir, los precios sombra permitidos tienen que ser razonables para el centro escogido como referente global.

La Tabla 3 muestra los resultados del análisis VEA. En primer lugar, puede destacarse la reducción en el número de centros considerados eficientes, que pasa de 19 a 13. Los 7 centros que desaparecen del recuento de centros totalmente eficientes estaban consiguiendo sus índices con valoraciones de los inputs y los outputs que serían inadecuadas para el centro número 3 , seleccionado como referente global. Por tanto, vemos

TABLA 3

ESTADÍSTICOS DESCRIPTIVOS DE LA EFICIENCIA VEA

\begin{tabular}{|l|c|c|c|c|c|}
\hline & Media & SD & Mín. & N eficiente & \% eficiente \\
\hline $\mathbf{E F}_{\text {CRS }}$ & 87,9 & 10,4 & 66,7 & 13 & 21,3 \\
$\mathbf{E F}_{\text {VRS }}$ & 93,7 & 8,1 & 72,2 & 26 & 42,6 \\
$\mathbf{E S}$ & 93,7 & 6,4 & 73,6 & 13 & 21,3 \\
\hline
\end{tabular}


que el poder discriminante se ha mejorado considerablemente imponiendo esta sencilla restricción. Por su parte, el número de centros eficientes desde el punto de vista de la gestión (frontera VRS) se redujo de 35 a 26, lo que supone una reducción del $26 \%$.

La Tabla 3 muestra algunas otras diferencias con respecto a la Tabla 2. Puede verse que la contribución a la ineficiencia global no se debe principalmente a la escala de operaciones, sino que se reparte por igual entre los problemas de escala y de gestión. En cualquier caso, puede mantenerse la conclusión de que el grado de eficiencia medio sigue siendo bastante elevado.

La Tabla 4 muestra cómo se ha reducido la disparidad en los precios sombra asignados por las diferentes unidades de la muestra, al incorporar la restricción del análisis VEA. Se muestra el porcentaje de variación de la desviación típica de cada uno de los inputs y outputs considerados. Solamente el input Recursos Humanos ha incrementado la disparidad de precios sombra en el análisis VEA. En el resto de las variables la desviación típica se ha reducido considerablemente. Los inputs de derivaciones $(-59.1 \%)$ y gastos corrientes $(-40,9)$ son los que más han reducido la dispersión de sus precios sombra. En el caso de los outputs son las visitas domiciliarias $(-40.6 \%)$ y el total de consultas (-30.3\%) las dimensiones que presentan una mayor reducción. Globalmente, se ha conseguido una reducción promedio en la desviación típica de los precios sombra de las dimensiones del $27,8 \%$. Esto indica una gran mejora en el grado de congruencia de las valoraciones realizadas de las actividades productivas de cada una de las unidades de la muestra en los programas matemáticos del análisis VEA.

Una vez estimados los índices de eficiencia VEA, hemos tratado de valorar la posible relación entre algunos factores contextuales y los índices obtenidos. Para ello, realizamos en primer lugar un análisis de regresión sobre el índice de eficiencia VEA de gestión $\left(\mathrm{EF}_{\mathrm{VRS}}\right)^{2}$, tomando como variables independientes el porcentaje de pobla-

\section{TABLA 4}

REDUCCIÓN (\%) DE LA DESVIACIÓN TÍPICA DE LOS PRECIOS SOMBRA CON VEA

\begin{tabular}{|c|c|c|c|}
\hline Inputs & Var \% & Outputs & Var \% \\
\hline Recursos Humanos & 10,3 & Total consultas & $-30,3$ \\
Gastos corrientes (C II) & $-40,9$ & Visitas domiciliarias & $-40,6$ \\
& & de enfermería & \\
Gastos de Farmacia (C IV) & $-32,4$ & Población*Cartera de servicios & $-9,4$ \\
Total derivaciones & $-59,1$ & Cumplimiento de Normas & $-19,8$ \\
& & Técnicas Mínimas & \\
\hline
\end{tabular}

2 También se realizaron análisis de regresión sobre el índice de eficiencia de escala, no encontrando ninguna relación significativa con estas variables explicativas, motivo por el que no se incluyen los resultados en el texto. 
ción anciana (ANC), el coeficiente de dispersión geográfica (G) y la presencia o no de docencia (MIR).

Se aprecia una clara relación negativa y significativa entre el porcentaje de población anciana y la eficiencia de gestión en los centros de salud analizados. A mayor porcentaje de población anciana cubierta por el centro, menor índice de eficiencia. Asimismo, se aprecia un efecto positivo de la dispersión geográfica y la ruralidad sobre la eficiencia de gestión. No se observa en cambio ninguna relación significativa entre la docencia MIR y la eficiencia medida a través del índice VEA.

Los resultados anteriores pueden ser puestos en duda por dos motivos. En primer lugar, puede existir una correlación bastante grande entre el valor $\mathrm{G}$ y el porcentaje de ancianos. De hecho, se comprobó que la correlación entre ambas variables alcanza el 57\%, siendo estadísticamente significativa al nivel 0,01. Por tanto, existe un problema de multicolinealidad en la regresión que dificulta la correcta valoración del efecto de cada variable sobre la variable dependiente. En segundo lugar, los índices DEA no se distribuyen ni independientemente ni normalmente, de manera que la metodología de análisis de regresión no resulta del todo adecuada. Por estos motivos, realizamos un segundo contraste, utilizando métodos no paramétricos para contrastar diferencias entre grupos, en lugar de un análisis de regresión conjunto.

En primer lugar, se contrastó la existencia de diferencias en la eficiencia media entre los centros con presencia MIR (1) y sin presencia MIR (0), mediante la prueba de rangos de Mann y Whitney. Los resultados se recogen en la Tabla 6. Se aprecia claramente que no existe una relación significativa entre la eficiencia de gestión y la presencia de MIR en los centros de salud.

La relación entre el valor $\mathrm{G}$ y el índice de eficiencia se contrastó mediante la prueba de Kruskall-Wallis para comprobar si existen diferencias significativas de eficiencia entre los 4 grupos de dispersión geográfica presentes en la muestra. En la tabla se aprecia que si bien los 5 centros con valor $\mathrm{G}=1$, tienen una menor eficiencia, las diferencias entre grupos no son estadísticamente significativas, con lo que no puede establecerse una relación entre la ineficiencia y el valor G.

Por último, para contrastar la relación entre eficiencia y porcentaje de población anciana, se realizaron 3 grupos en función del índice de eficiencia. El grupo 1 está formado por los 18 centros de salud cuya eficiencia es inferior al 91\%. El grupo 21 lo componen los 17 centros ineficientes pero con índice de eficiencia mayor al 91\%. Por último, el grupo 3 lo componen las 26 unidades eficientes. La Tabla 8 contiene los resultados del test de rangos de Kruskal-Wallis. Se aprecia una población significativamente más envejecida en el grupo 1 de menor eficiencia.

\section{Conclusiones}

Los resultados de este trabajo muestran, nuevamente, el déficit de capacidad discriminante de la metodología de Análisis Envolvente de Datos (DEA) cuando 
TABLA 5

FACTORES RELACIONADOS CON LA INEFICIENCIA DE GESTIÓN (EF VRS $)$

\begin{tabular}{|l|c|c|c|}
\hline & Coeficiente & Coef. Estand. & $\mathbf{t}$ \\
\cline { 2 - 4 } Constante & 1,000 & & $25,8^{* * *}$ \\
ANC & $-0,006$ & $-0,46$ & $-3,08^{* * *}$ \\
G & 0,022 & 0,26 & $1,78^{*}$ \\
MIR & 0,013 & 0,08 & 0,63 \\
$R^{2}$ & 0,156 & & \\
\hline
\end{tabular}

*** Nivel de significación 0,01** Nivel de significación 0,05* Nivel de significación 0,1

TABLA 6

RELACIÓN ENTRE EFICIENCIA Y DOCENCIA MIR

\begin{tabular}{|l|c|c|}
\hline & N & Rango promedio \\
\cline { 2 - 3 } Sin docencia MIR & 34 & 29,87 \\
Con docencia MIR & 27 & 32,43 \\
U de Mann-Whitney & 420,5 & \\
$\mathrm{p}$ & 0,56 & \\
\hline
\end{tabular}

TABLA 7

RELACIÓN ENTRE EFICIENCIA Y DISPERSIÓN GEOGRÁFICA

\begin{tabular}{|l|c|c|}
\hline & N & Rango promedio \\
\cline { 2 - 3 } $\mathrm{G}=1$ & 5 & 25,90 \\
$\mathrm{G}=2$ & 22 & 30,30 \\
$\mathrm{G}=3$ & 14 & 34,96 \\
$\mathrm{G}=4$ & 20 & 30,28 \\
$\mathrm{H}$ de Kruskall-Wallis & 1,28 & \\
$\mathrm{p}$ & 0,73 & \\
\hline
\end{tabular}

TABLA 8

RELACIÓN ENTRE EFICIENCIA Y PORCENTAJE DE POBLACIÓN ANCIANA

\begin{tabular}{|l|c|c|}
\hline \multirow{2}{*}{ Grupo eficiencia } & N & Rango promedio \\
\cline { 2 - 3 } 1 & 18 & 38,06 \\
2 & 17 & 24,24 \\
3 & 26 & 30,54 \\
H de Kruskal-Wallis & 5,33 & \\
$\mathrm{p}$ & 0,07 & \\
\hline
\end{tabular}


se tienen en cuenta múltiples inputs y outputs con una muestra de tamaño limitado. Existen al menos tres maneras de mejorar la capacidad discriminante del DEA. La más sencilla es reducir el número de dimensiones (input-output) consideradas. En este caso, se mejora la capacidad discriminante pero se pierde información relevante para realizar la discriminación. En segundo lugar, incrementar el tamaño de la muestra. Desgraciadamente esta solución no es factible cuando se trabaja con poblaciones enteras de tamaño reducido, como es el caso de muchos estudios empíricos y, concretamente, el de este trabajo. Una tercera aproximación consiste en mejorar el poder de discriminación de la propia técnica introduciendo información adicional que deba tenerse en cuenta para realizar la discriminación. El Value Efficiency Analuysis (VEA) permite incorporar fácilmente información cualitativa, como es la elección de un centro ideal como referencia en base a la opinión de los expertos o a partir del análisis previo con DEA, mejorando sensiblemente el poder discriminante del DEA.

En este trabajo se han aplicado ambas metodologías (DEA y VEA) a una muestra 61 de centros de salud asturianos durante el año 2002. La muestra incluye todos los centros de salud de Asturias que cumplen ciertos criterios de homogeneidad para ser incluidos en el estudio. Por tanto, en este estudio no es posible incrementar el poder discriminante del DEA aumentando el tamaño de la muestra. Ateniéndonos a los resultados DEA, los centros de salud evaluados tienen una eficiencia técnica alta, siendo mayor la ineficiencia debida a la escala que a la gestión. El análisis muestra que la ineficiencia de escala se debe fundamentalmente a factores relacionados con los centros de salud de mayor tamaño. La dimensión de los centros es una circunstancia crítica, a juzgar por nuestros resultados, y que no siempre se tiene en cuenta en la planificación sanitaria de estas estructuras.

El análisis VEA sigue mostrando unos niveles de eficiencia bastante altos, pero los atribuye por igual a las causas relacionadas con la escala y las debidas a problemas de gestión. Incorporando solamente la información sobre un centro de salud que puede considerarse como un buen referente general, el análisis VEA mejora el poder discriminante sensiblemente, pasando de 19 centros eficientes con DEA a 13 con VEA. En realidad el análisis VEA permite descubrir de manera sencilla aquellos centros de salud que «salen» eficientes en el DEA «sin serlo», simplemente a causa del extremado carácter conservador de dicha técnica. El VEA no permite que un centro de salud sea considerado eficiente simplemente por ser el que utiliza la menor cantidad de un input o ser el que obtiene la cantidad mayor de algún output. El comportamiento debe ser aceptable globalmente al compararse con el centro de referencia, siendo este el motivo por el que se consigue mejorar la deficiencia de poder discriminante del DEA. De hecho, en este estudio se ha constatado una disminución promedio del $28 \%$ en la dispersión de los precios sombra asignados por las distintas unidades de la muestra a cada una de las dimensiones de input y output. Por tanto, el mayor poder discriminante viene acompañado de una mayor racionalidad en la asignación de precios sombra en los programas matemáticos del análisis VEA. 
Respecto a los factores de contexto, se observa una clara relación positiva y significativa con el porcentaje de población anciana cubierta por el centro. Este hallazgo ya ha sido objetivado por otras vías como la observación de un incremento en el consumo y gasto de farmacia relacionado con el incremento de la edad (Goñi 1999). Por el contrario, aunque se aprecia cierta relación con la dispersión geográfica de la población, no puede concluirse que dicha relación sea estadísticamente significativa, al existir problemas de multicolinealidad con el porcentaje de población anciana. Finalmente, no se ha encontrado relación alguna entre los valores de la eficiencia y la existencia de docencia MIR.

Es necesario continuar investigando la forma de incrementar la capacidad discriminante de estos métodos, con el fin de que no pierdan su operatividad a causa de su principal virtud, es decir, la obtención de un índice mediante la inclusión de múltiples inputs y ouputs. Para ello, en el futuro esperamos validar este método utilizando un ranking confeccionado por un conjunto de expertos y comparándolo con distintos rankings obtenidos mediante DEA, VEA y métodos de frontera estocástica.

\section{Referencias}

[1] ALLEN R., ATHANASSOPOULOS, R., DYSON G. y E. THANASSOULIS (1997), «Weights Restrictions and Value Judgements in Data Envelopment Analysis: Evolution, Development and Future Directions», Annals of Operations Research, 73, 13-34.

[2] ALI, A.I. (1994), « Computational aspects of Data Envelopment Analysis», en DEA. Theory, Methodology and Applications (Charnes, Cooper, Lewin y Seiford, Eds.), Kluwer Academic Publishers, Boston.

[3] ALONSO R., GARCÍA R. y J.A. GONZÁLEZ (1995), «La Cartera de Servicios en Atención Primaria», Medifam, 3, 131-136.

[4] BANKER R.D., CHARNES A. y W.W. COOPER (1984), «Some Models for Estimating Technical and Scale Inefficiencies», Management Science, 39, 1261-1264.

[5] CHARNES A., COOPER W.W. y E. RHODES (1978), «Measuring the Efficiency on Decision Making Units», European Journal of Operational Research, 2, 429-44.

[6] DYSON, G. y E. THANASSOULIS (1988), «Reducing Weight Flexibility in Data Envelopment Analysis», Journal of Operational Research Society, 6, 563-576.

[7] FUENTELSAZ L., MARCUELLO C. y O. URBINA (1996), «Eficiencia productiva en la prestación de servicios de salud: Una aplicación a los Centros de Atención Primaria», Hacienda Pública Española, 138: 29-39.

[8] GARCÍA, R., ALONSO, R., LÓPEZ, E., GONZÁLEZ-POSADAS, JA. y R. SEMPERE (1997) «Estrategia de desarrollo de la cartera de servicios», Revista de Administración Sanitaria, 1(4), 595-610.

[9] GARCÍA F., MARCUELLO C., SERRANO D. y O. URBINA (1999), «Evaluation of Efficiency in Primary Health Care Centres: An application of Data Envelopment Analysis», Financial Accountability and Management, 15, 1:67-83 
[10] GARCÍA F., MARCUELLO C., SERRANO D. y O. URBINA (1996), «Evaluación de la eficiencia en centros de atención primaria. Una aplicación del análisis envolvente de datos», Revista Española de Salud Publica, 70, 211-220.

[11] GARCÍA, L. y S. MINUÉ (1998), «La medida del producto de atención primaria», Cuadernos de Gestión, 4(4), 191-200.

[12] GARCÍA J.V. y E. GONZÁLEZ (2004), «Aplicación y utilidad del análisis envolvente de datos en la medida de la eficiencia de los equipos de atención primaria de Asturias», XXIV Jornadas de Economía de la Salud, El Escorial.

[13] GARCÍA MJ., BOLÍVAR B., ROSELL M., ROSET M. y S. JUNCOSA (1998), «Adaptación de los Ambulatory Care Groups (ACG) a la Clasificación Internacional de Atención Primaria (CIAP)», Gaceta Sanitaria, 12, supl 1, 19.

[14] GOÑI, S. (1998), «El análisis envolvente de datos como sistema de evaluación de la eficiencia técnica de las organizaciones del sector público: Aplicación en los Equipos de Atención Primaria», Revista Española de Financiación y Contabilidad, 97, 979-1004.

[15] GOÑI, S. (1999), «An analysis of the effectiveness of Spanish primary health care teams», Health Policy, 48, 107-117.

[16] HALME M., JORO T., KORHONEN P., SALO S. y J. WALLENIUS (1999), «A Value Efficiency Approach to Incorporating Preference Information in Data Envelopment Analysis», Management Science, 45(1), 103-115.

[17] INSALUD, Subdirección General de Coordinación Administrativa (1997), «Cartera de servicios de Atención Primaria. Definiciones, criterios de acreditación, indicadores de cobertura y normas técnicas mínimas». Madrid.

[18] JUNCOSA S., BOLÍVAR B., ROSET M. y R. TOMÁS (1999), «Performance of an ambulatory casemix measurement system in primary care in Spain», European Journal of Public Health, 9(1): 27-35.

[19] MARTÍ, T. y V. GRENZNER (1999), «Modelos de Atención Primaria de Catalunya», Cuadernos de Gestión en Atención Primaria, 5(3): 116-123.

[20] ORUETA J., LÓPEZ, J., BÁEZ K., AIARZAGUENA, JM., ARANGUREN, J. y E. PEDRERO (1999), «Application of the Ambulatory Care Groups in the primary care of a European national health care system: Does it work?», Medical Care, 37(3):524-554

[21] PEDRAJA F., SALINAS J. y P. SMITH (1997), «On the Role of Weight Restrictions in Data Envelopment Analysis», Journal of Productivity Analysis, 8, 215 230.

[22] PINILlOS M. y F. ANTOÑANZAS (2002), «La Atención Primaria de Salud: descentralización y eficiencia», Gaceta Sanitaria, 16(5): 401-407.

[23] PINILLOS, M. (2003). Eficiencia del servicio público de atención sanitaria primaria. Papeles de Economía Española 95: 213-225.

[24] PUIG, J. (2000), «Eficiencia en la atención primaria de salud: una revisión crítica de las medidas de frontera», Revista Española de Salud Pública, 74: 483-495.

[25] PUIG, J. y E. DALMAU (2000), «¿Qué sabemos acerca de la eficiencia de las organizaciones sanitarias en España?. Una revisión de la literatura económica», XX Jornadas de Economía de la Salud, AES, Palma de Mallorca.

[26] PUJOL RIBERA E., GENÉ BADIA J., SANS CORRALES M., SAMPIETRO-COLOM L., PASARÍN RUA M.I., IGLESIAS-PÉREZ B., CASAJUANA-BRUNET J., 
y G. ESCARAMÍS-BABIANO (2006), «El producto de la atención primaria definido por profesionales y usuarios», Gaceta Sanitaria, 20(3): 209-219.

[27] ROLL Y., COOK W.D. y B. GOLANY (1991), «Controlling Factor Weights in Data Envelopment Analysis», IIE Transactions, 23, 2-9.

[28] URBINA, O. y D. SERRANO (1997). Evaluación de la eficiencia médica en atención primaria, XVII Jornadas de Economía de la Salud, AES, Murcia.

[29] WONG Y-H.B. y J.E. BEASLEY (1990), «Restricting Weight Flexibility in Data Envelopment Analysis», Journal of Operational Research Society, 41, 829-835. 
